\title{
Lower-extremity arterial stiffness vs. aortic stiffness in the general population
}

\author{
Peter Wohlfahrt ${ }^{1,2,6}$, Alena Krajčoviechová ${ }^{1}$, Jitka Seidlerovái, Markéta Galovcová ${ }^{2}$, Jan Bruthans ${ }^{2}$, \\ Jan Filipovský ${ }^{3}$, Stéphane Laurent ${ }^{4}$ and Renata Cífková ${ }^{1,2,5,6}$
}

While determinants of aortic pulse wave velocity (aPWV) are well known, much less is known about factors affecting lower-extremity pulse wave velocity (lePWV). Unlike aPWV, increased lePWV does not predict cardiovascular risk, but limits lower-extremity blood flow and is associated with increased left ventricular mass. The aim of this study was to compare the effect of cardiovascular risk factors on aPWV and lePWV. A total of 911 individuals from the Czech post-MONICA study (a randomly selected $1 \%$ representative population sample, mean age $54 \pm 13.5$ years, $47 \%$ men) were examined. Pulse wave velocity was measured using the SphygmoCor device. Aging had a large effect on aPWV, but only a small effect on lePWV. After adjustment for covariates, we observed that hypertension, diabetes, chronic kidney disease and dyslipidemia were positively and significantly associated with aPWV. However, only hypertension had a significant effect on lePWV. Increased ankle systolic blood pressure was associated with increased aPWV independently of brachial blood pressure. Ankle systolic blood pressure was more closely related to aPWV than lePWV. Subjects with an ankle-brachial index $<1.0$ had higher aPWV and lower lePWV compared with individuals with a normal ankle-brachial index. Lower-extremity arterial stiffness is affected by age and cardiovascular risk factors to a lesser extent than aortic stiffness. Increased ankle systolic blood pressure is linked not only to increased lower-extremity arterial stiffness, but also increased aortic stiffness. In subjects with a low ankle-brachial index, lower-extremity arterial stiffness is spuriously decreased.

Hypertension Research (2013) 36, 718-724; doi:10.1038/hr.2013.21; published online 11 April 2013

Keywords: ankle blood pressure; arterial stiffness; general population; peripheral arterial disease; pulse wave velocity

\section{INTRODUCTION}

Aortic pulse wave velocity (aPWV) as a measure of aortic stiffness is an independent predictive factor for all-cause and cardiovascular morbidity and mortality in patients with various levels of cardiovascular risk. ${ }^{1}$ In contrast, stiffness of other arterial territories has a smaller or no ability to predict cardiovascular outcomes. ${ }^{2-6}$ Indeed, carotid stiffness has been shown to predict cardiovascular events in patients with end-stage renal disease ${ }^{2}$ and after renal transplantation. ${ }^{3}$ However, in patients with manifest cardiovascular disease, carotid stiffness was not an independent risk factor for vascular events. ${ }^{4}$ Brachial and femoro-tibial PWV were not predictors of cardiovascular mortality in patients with end-stage renal disease. ${ }^{5}$ Furthermore, only carotid-femoral PWV was independently associated with coronary artery calcification, carotid and femoral plaques, whereas carotidradial and femoro-tibial PWV were not. ${ }^{6}$

On the other hand, femoral artery stiffness was found to be closely associated with symptoms of lower limb peripheral arterial disease
(PAD). ${ }^{7}$ Furthermore, a negative correlation was shown between brachial-ankle PWV and blood flow in the popliteal artery, ${ }^{8}$ and between femoral arterial stiffness and foot transcutaneous oxygen tension. ${ }^{9}$ Lately, increased lower-extremity PWV (lePWV) determined by a high ankle-brachial index (ABI) was found to be linked to greater left ventricular (LV) mass through nonatherosclerotic pathways. ${ }^{10}$

Despite these findings suggesting the importance of lowerextremity arterial stiffness in lower-extremity blood flow and its association with LV hypertrophy, there have been only a few studies identifying determinants of lower-extremity arterial stiffness. Most of these studies determined lower-extremity arterial stiffness from local femoral arterial distensibility. Measurement of local vascular distensibility is often confounded by pressure most often measured at the brachial level and not locally, resulting in amplification-related errors. In addition, previous studies have reported the effect of lowerextremity peripheral arterial disease (PAD) on brachial-ankle PWV,

${ }^{1}$ Center for Cardiovascular Prevention of the First Faculty of Medicine, Charles University and Thomayer University Hospital, Prague, Czech Republic; ${ }^{2}$ Department of Preventive Cardiology, Institute for Clinical and Experimental Medicine, Prague, Czech Republic; ${ }^{2}$ 2nd Department of Internal Medicine, Center for Hypertension, Charles University, Pilsen, Czech Republic; ${ }^{4}$ Department of Pharmacology, Georges Pompidou European Hospital, INSERM U970 and Paris University Descartes, Paris, France; ${ }^{5}$ Department of Cardiology and Angiology, First Faculty of Medicine, Charles University, Prague, Czech Republic and ${ }^{6}$ International Clinical Research Center, St Ann's University Hospital, Brno, Czech Republic

Correspondence: Dr P Wohlfahrt, Center for Cardiovascular Prevention, Thomayer Hospital, Videnska 800, 14059 Prague, Czech Republic.

E-mail:wohlfp@gmail.com

Received 4 September 2012; revised 13 December 2012; accepted 26 December 2012; published online 11 April 2013 
but no study has determined the effect of lower-extremity arterial stenosis on lePWV.

The aim of the present study was to compare the effect of cardiovascular risk factors on lower-extremity and aortic stiffness. In order to determine the effect of lower-extremity peripheral arterial disease on lower-extremity arterial stiffness, lePWV was compared in patients with a low, normal and high ankle-brachial index.

\section{METHODS}

Study population

The Czech post-MONICA study is a population survey studying trends and determinants of cardiovascular risk factors in a random sample of the Czech population. Methods of the Czech post-MONICA study have been described elsewhere. ${ }^{11}$ Our study included patients aged $>25$ years, residing in Pilsen district. The response rate in this district was $68 \%$. A total of 911 patients ( $98 \%$ of patients examined in Pilsen district) had complete data on ABI and PWV. The study was approved by the local ethics committee of the Institute for Clinical and Experimental Medicine and Thomayer University Hospital, and was in accordance with the Declaration of Helsinki.

Hypertension was defined as systolic blood pressure (SBP) $\geqslant 140 \mathrm{~mm} \mathrm{Hg}$ and/or diastolic blood pressure (DBP) $\geqslant 90 \mathrm{mmHg}$, or current use of antihypertensive medication. Diabetes was defined as fasting plasma glucose $\geqslant 7.0 \mathrm{mmoll}^{-1}$ or use of oral antidiabetic drugs and/or insulin. Dyslipidemia was defined as total cholesterol $\geqslant 5 \mathrm{mmoll}^{-1}$ or high density lipoproteincholesterol $<1 \mathrm{mmoll}^{-1}$ in men and $<1.2 \mathrm{mmoll}^{-1}$ in women or use of lipid-lowering drugs. Chronic kidney disease (CKD) was defined as estimated glomerular filtration rate (eGFR) $<60 \mathrm{ml} \mathrm{min}^{-1}$ per $1.73 \mathrm{~m}^{2}$. Glomerular filtration rate was estimated using the simplified CKD-EPI formula as described by Levey. ${ }^{12}$

\section{Measurement of large artery properties}

Large artery properties were measured using the SphygmoCor device (AtCor Medical Ltd, West Ryde, New South Wales, Australia) in the recumbent position as reported before. ${ }^{13}$ Aortic pulse wave velocity and lePWV were assessed according to recommendations. ${ }^{14}$ Consecutive registrations of the pulse waves are ECG-gated and thus, the time shift $(\Delta t)$ between the foot of wave at the first and second sites can be calculated. The distance between the two sites was measured on the body surface. To determine aPWV, we measured the distance from the jugular fossa to the pulsation of the femoral artery in the groin and subtracted the distance from the jugular fossa to carotid pulsation in order to obtain the traveled distance $(D)$. The distance between the femoral artery and dorsal pedal/posterior tibial arteries was measured to calculate lePWV. PWV was calculated as $D(\mathrm{~m}) / \Delta t(\mathrm{~s})$.

\section{Doppler ABI measurement}

Details of ABI measurement are described in our previous paper. ${ }^{15}$ Briefly, appropriately sized cuffs of a mercury sphygmomanometer (Baumanometer TM, WA Baum, New York, NY, USA) were placed proximal to the ankles and on the right arm. After a 5 min resting period, SBP was measured on the right brachial artery, right dorsal pedal and posterior tibial arteries, left dorsal pedal and tibial arteries (in this order) using a pocket Doppler device with an $8 \mathrm{MHz}$ probe (DopplexmultiTM, Huntleigh, Cardiff, UK). All measurements were performed by two physicians experienced in ABI measurement. ABI was calculated separately for each leg by dividing the highest of the ankle systolic pressures by the brachial systolic pressure. The lower of the two leg ABI values was used in further analysis. Systolic blood pressure measured on the right posterior tibial artery was used for further analyses and is indicated as ankle blood pressure (aSBP). A cutoff value of $175 \mathrm{~mm} \mathrm{Hg}$ was used to identify subjects with increased aSBP as reported earlier. ${ }^{16}$

\section{Statistical analysis}

Descriptive statistics are given as mean \pm s.d., mean (95\% CI) or frequency and percent. In the figures, error bars represent s.e.m. In order to determine the effect of age and gender on arterial stiffness, analysis of covariance with adjustment for mean arterial pressure (MAP) was performed while subdividing individuals into quintiles of age. The effect of cardiovascular risk factors on arterial stiffness was determined by analysis of covariance with adjustment for age, gender and MAP. Partial eta-squared $\left(\eta^{2}\right)$ is reported, indicating how much variability of the dependent variable can be predicted by the independent variable. The effect size was considered large for $\eta_{\mathrm{p}}^{2}>0.30$. To determine the effect of PAD on arterial stiffness, participants were divided into three groups on the basis of their $\mathrm{ABI}$ : low $(\mathrm{ABI}<1.0)$, normal $(\mathrm{ABI}=1-1.4)$, and high $(\mathrm{ABI} \geqslant 1.4)$. Arterial properties between these groups were compared using one-way analysis of variance with Turkey's post-hoc test. Differences between groups were reassessed after adjustment for age, gender and MAP. Determinants of aPWV, lePWV and aSBP were assessed by multiple linear regression analysis. Variables significantly associated with parameters of arterial stiffness in univariate analyses were included into the multiple regression analyses. Standardized values of the mean and the systolic blood pressure were used to decrease colinearity between parameters. We considered the stability of the regression model to be disturbed by multi-colinearity if the tolerance was $<0.1$. Standardized beta is reported $\left(B_{\mathrm{S}}\right)$. A $\beta_{\mathrm{S}}$ of 0.1 indicates that when the independent variable increases by 1 s.d., the dependent variable increases by 0.1 s.d. All calculations were performed using SPSS 16 software (SPSS, Chicago, IL, USA). A two-sided $P$-value $<0.05$ was considered to be statistically significant.

\section{RESULTS}

\section{Study population characteristics}

Characteristics of the study population of 911 individuals (430 male and 481 female; mean age of $54.1 \pm 13.49$ years), with complete data, are provided in Table 1.

\section{Effect of age and gender on aortic and lower-extremity pulse wave velocities}

Aortic pulse wave velocity increased with age $(P<0.001)$ both in men and women, with the mean aPWV higher in men (Figure 1). Partial eta-squared representing explained variability was high for age $\left(\eta_{\mathrm{p}}^{2}=0.32\right)$, while there was only a minor effect of gender $\left(\eta^{2} \mathrm{p}=0.01\right)$. No interaction between age and gender was found $(P=0.68)$.

Mean lower-extremity pulse wave velocity was higher in men than in women and showed a significant increase with age. There was a

\section{Table 1 Study population characteristics}

\begin{tabular}{|c|c|}
\hline $\mathrm{SBP}, \mathrm{mm} \mathrm{Hg}$ & $127.70 \pm 17.15$ \\
\hline $\mathrm{DBP}, \mathrm{mm} \mathrm{Hg}$ & $69.48 \pm 10.81$ \\
\hline MAP, $\mathrm{mm} \mathrm{Hg}$ & $92.77 \pm 9.81$ \\
\hline lePWV, $\mathrm{ms}^{-1}$ & $9.77 \pm 1.81$ \\
\hline aPWV, $\mathrm{ms}^{-1}$ & $8.42 \pm 2.34$ \\
\hline eGFR, $\mathrm{ml} \mathrm{min}-1$ per $1.73 \mathrm{~m}^{2}$ & $75.08 \pm 12.46$ \\
\hline Total cholesterol, mmoll-1 & $5.16 \pm 1.01$ \\
\hline Triglycerides, mmoll-1 & $1.51 \pm 1.03$ \\
\hline HDL-cholesterol, mmoll-1 & $1.43 \pm 0.42$ \\
\hline LDL cholesterol, mmol ${ }^{-1}$ & $2.92 \pm 1.28$ \\
\hline Fasting plasma glucose, $\mathrm{mmoll}^{-1}$ & $5.39 \pm 1.23$ \\
\hline $\mathrm{CHD}, n(\%)$ & $46(5 \%)$ \\
\hline Hypertension, $n(\%)$ & $420(46 \%)$ \\
\hline Diabetes, $n(\%)$ & $70(8 \%)$ \\
\hline Dyslipidemia, $n(\%)$ & $640(71 \%)$ \\
\hline Current smokers, $n(\%)$ & $254(28 \%)$ \\
\hline Low $\mathrm{ABI}, n(\%)$ & $28(3 \%)$ \\
\hline High $\mathrm{ABI}, n(\%)$ & $23(2.5 \%)$ \\
\hline
\end{tabular}

Abbreviations: $A B I$, ankle-brachial index; aPWV, aortic pulse wave velocity; $C H D$, coronary heart disease; DBP, diastolic blood pressure; eGFR, estimated glomerular filtration rate; $\mathrm{HDL}$, high density lipoprotein; LDL, lowdensity lipoprotein; lePWV, lower-extremity pulse wave velocity; MAP, mean arterial pressure; SBP, brachial systolic blood pressure. MAP, mean arterial pressure; SBP, brachial systolic blood pressure.
Values are presented as mean \pm standard deviation or frequency (percent). 


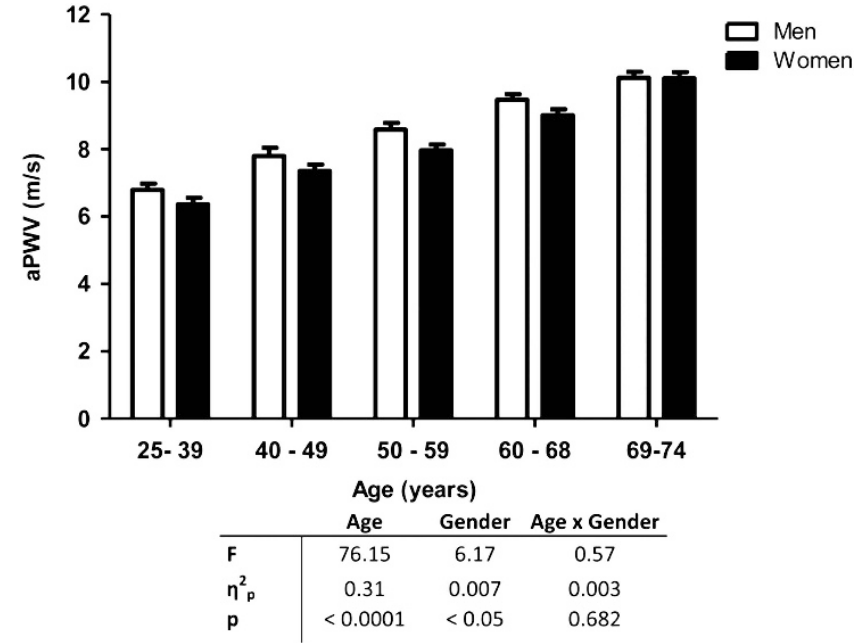

Figure 1 Effect of age and gender on aortic PWV (aPWV). Data adjusted for mean arterial pressure, height and weight.



Figure 2 Effect of age and gender on lower-extremity PWV (lePWV). Data adjusted for mean arterial pressure, height and weight.

significant age-gender interaction. While, in women, there was a linear increase in lePWV with increasing age $(P<0.001$ for linear term), in men there was only a minor change after 59 years of age. Partial eta squared was higher for age $\left(\eta_{\mathrm{p}}^{2}=0.054\right)$ than for gender $\left(\eta_{\mathrm{p}}^{2}=0.026\right)$, with only a minor effect of age-gender interaction $\left(\eta^{2} \mathrm{p}=0.013\right)$ (Figure 2). When individuals with low ABI were excluded from the analysis of age and gender effect on lePWV, the age-gender interaction was no longer significant $(P=0.12)$, while the $\eta_{\mathrm{p}}^{2}$ for age and gender increased.

\section{Effect of cardiovascular risk factors on aPWV and lePWV}

Table 2 summarizes the effect of different cardiovascular risk factors on aPWV and lePWV. In unadjusted analyses, subjects with hypertension, diabetes and dyslipidemia had higher aPWV. The effect of these risk factors on aPWV remained unchanged after adjustment for covariates. Lower-extremity arterial stiffness was higher in individuals with hypertension and dyslipidemia. However, after adjustment, only hypertension remained associated with elevated lePWV. The effect of cardiovascular risk factors on lePWV did not change significantly after excluding individuals with low ABI.

\section{Association of aPWV and lePWV with lower-extremity peripheral arterial disease}

Aortic pulse wave velocity was higher in subjects with low and high $\mathrm{ABI}$ compared with the normal $\mathrm{ABI}$ group (high $\mathrm{ABI}, 11.1 \pm 2.8$ vs. $8.3 \pm 2.3, P<0.0001$; low ABI, $10.8 \pm 2.5$ vs. $8.3 \pm 2.3, P<0.0001$, respectively). There was no difference in aPWV between patients with low and high ABI $(P=0.86)$. The elevation of aPWV in both extreme $\mathrm{ABI}$ groups remained unchanged after adjustment for age, gender and MAP (Figure 3a).

There was a linear increase in lePWV with increasing $\mathrm{ABI}$ $(P<0.001$ for linear trend). Lower-extremity PWV was lowest in individuals in the low ABI group $(8.82 \pm 1.85)$, intermediate in individuals with normal $\mathrm{ABI}(9.78 \pm 1.81)$ and highest in individuals with high ABI (10.98 \pm 1.28$)$. In a model adjusted for age, gender and MAP, individuals with low ABI had lower lePWV compared with the normal ABI group (8.23 (7.57-8.89) vs. 9.81 (9.70-9.92), $P<0.0001$ ) and the high ABI group (8.23 (7.57-8.89) vs. 10.24 (9.55-10.93, $P<0.0001)$, while there was no difference between the normal and high $\mathrm{ABI}$ groups (Figure $3 \mathrm{~b}$ ).

\section{Determinants of aPWV and lePWV}

In univariate analyses, the following parameters were associated with aPWV: age, MAP, SBP, DBP, serum cholesterol, waist circumference, triglycerides, glucose, creatinine level, eGFR, waist circumference, lePWV, heart rate and aSBP. In a multivariate model of linear regression (Table 3), age had the largest effect on aPWV, explaining $36 \%$ of the total variance. Among the conventional cardiovascular risk factors, MAP, SBP, abdominal obesity and CKD were independently associated with aortic stiffness. Lower-extremity SBP was also associated with aPWV independently of brachial SBP and other cardiovascular risk factors. Individuals with aSBP $>175 \mathrm{~mm} \mathrm{Hg}$ had higher aPWV at all levels of brachial blood pressure (Figure 4), which suggests that ankle systolic blood pressure is associated with aortic stiffness independently of brachial systolic blood pressure. In the receiver operating characteristic analysis (area under the curve $=0.71 \pm 0.02, P<0.001$ ), after excluding individuals with low ABI, aSBP $>175 \mathrm{~mm} \mathrm{Hg}$ had a sensitivity of $58 \%$ and a specificity of $84 \%$ to detect subjects with aPWV $>10 \mathrm{~m} \mathrm{~s}^{-1}$.

In univariate analyses, the following parameters were associated with lePWV: SBP, DBP, MAP, aSBP, age, weight, hip circumference, eGFR, serum creatinine, aPWV and glucose levels. In the multivariate model of linear regression (Table 3), aSBP, age, gender, MAP, low ABI and hip circumference were independent predictors of lePWV.

In multiple regression analyses, after excluding individuals with low ABI, ankle blood pressure was determined by brachial SBP, MAP, aPWV, height, age and lePWV. Aortic stiffness $\left(B_{S}=0.11, P<0.001\right.$, $R^{2}=0.02$ ) had a larger effect on ankle blood pressure than lePWV $\left(ß_{\mathrm{S}}=0.06, P<0.001, R^{2}=0.004\right)$.

\section{DISCUSSION}

In this study, we compared the determinants of aortic stiffness with those of lower-extremity arterial stiffness. We observed that the effect of cardiovascular risk factors on arterial stiffness differed between these two arterial territories. The principal findings of our study are as follows: (1) age and cardiovascular risk factors such as hypertension, 
Table 2 Effect of cardiovascular risk factors on aortic and lower-extremity PWV

\begin{tabular}{|c|c|c|c|c|}
\hline & Unadjusted aPWV & Adjusted aPWV & Unadjusted lePWV & Adjusted lePWV \\
\hline Yes & $9.70(9.47-9.92)$ & $8.99(8.81-9.18)$ & $10.22(10.04-10.41)$ & $9.95(9.77-10.13)$ \\
\hline$P$ & $<0.001$ & $<0.001$ & $<0.001$ & 0.018 \\
\hline$\eta_{p}^{2}$ & 0.253 & 0.071 & 0.05 & 0.006 \\
\hline No & 8.24 (8.09-8.39) & $8.36(8.25-8.48)$ & $9.75(9.62-9.87)$ & $9.79(9.69-9.91)$ \\
\hline$P$ & $<0.001$ & $<0.001$ & 0.30 & 0.08 \\
\hline$\eta_{\mathrm{p}}^{2}$ & 0.059 & 0.015 & - & - \\
\hline \multicolumn{5}{|c|}{ Dyslipidemia $^{\mathrm{b}}$} \\
\hline Yes & $8.74(8.56-8.91)$ & $8.53(8.40-8.66)$ & 9.89 (9.75-10.03) & $9.87(9.62-10.12)$ \\
\hline \multicolumn{5}{|c|}{ Current smoking ${ }^{\mathrm{b}}$} \\
\hline Yes & $7.95(7.68-8.22)$ & $8.23(7.99-8.47)$ & 9.74 (9.49-9.99) & $9.83(9.62-9.88)$ \\
\hline No & $8.59(8.24-8.79)$ & $8.49(8.36-8.62)$ & $9.77(9.54-10.01)$ & $9.75(9.60-10.06)$ \\
\hline$P$ & $<0.001$ & 0.06 & 0.98 & 0.53 \\
\hline$\eta^{2} p$ & 0.014 & - & - & - \\
\hline \multicolumn{5}{|c|}{ Chronic kidney disease ${ }^{\mathrm{b}}$} \\
\hline Yes & $10.38(9.56-10.77)$ & $9.19(8.79-9.58)$ & $9.97(9.54-10.40)$ & $9.55(9.16-9.94)$ \\
\hline No & 8.25 (8.09-8.39) & $8.35(8.23-8.47)$ & $9.75(9.63-9.88)$ & $9.79(9.68-9.91)$ \\
\hline$P$ & $<0.001$ & $<0.001$ & 0.35 & 0.23 \\
\hline$\eta^{2} p$ & 0.064 & 0.017 & - & - \\
\hline
\end{tabular}

Abbreviations: aPWV, aortic pulse wave velocity; lePWV, lower-extremity pulse wave velocity.

adjusted for age and gender.

${ }^{\mathrm{b}}$ Adjusted for age, gender and mean arterial pressure.

Data are expressed as mean $(95 \% \mathrm{Cl})$.

Significant $P$-values are indicated in bold

diabetes, dyslipidemia and chronic kidney disease affect aortic stiffness, while only age and hypertension have a significant effect on lower-extremity stiffness, (2) abdominal obesity increases aortic stiffness, while a larger hip circumference is associated with lowerextremity stiffness, (3) increased ankle systolic blood pressure is associated with increased aortic stiffness independently of brachial systolic pressure and other cardiovascular risk factors, (4) lowerextremity arterial stiffness is spuriously decreased in individuals with lower-extremity PAD.

In our study, age had a major effect on aortic stiffness, but only a small effect on lower-extremity arterial stiffness. This may be due to the different histological structure of these arteries and a different effect of aging on these structures. In elastic arteries, aging leads to fragmentation and alteration of the elastic fiber network responsible for buffering function, while aging in the muscular arteries leads to changes in the extracellular matrix involving mainly collagen fibers, and to hypertrophy of vascular smooth muscle cells and the arterial wall, acting in the opposite direction on arterial stiffness. ${ }^{17,18}$ Indeed, there is general agreement that aortic stiffness increases with age, but there are discrepant data on aging of the lower-extremity arteries. While, in some studies, ${ }^{19,20}$ no increase in femoral artery stiffness with age was found, there was some increase in femoral ${ }^{21}$ and lower limb arterial stiffness ${ }^{22-24}$ in others. This discrepancy can be explained by the small effect of age on lePWV and different methods of arterial stiffness measurement.

We found hypertension to increase lower-extremity arterial stiffness. However, in other studies, ${ }^{25,26}$ hypertension had no effect or it decreased brachial artery stiffness. Of note, the brachial artery is also less prone to atherosclerosis than major arteries in the lower extremities. This suggests that differences in muscular arteries do exist.

We found a significant gender difference in aPWV and lePWV that was independent of anthropometric parameters and blood pressure. The effect of gender on stiffness was small as evident from the small $\eta^{2}$. We found a significant age-gender interaction on lePWV that became non-significant after excluding individuals with PAD. This was probably due to the higher prevalence of men with PAD. The gender difference in lePWV observed in our study is in agreement with previous reports. ${ }^{20,27,28}$

In our study, abdominal obesity expressed as waist circumference was associated with increased aPWV. Waist circumference is closely associated with visceral fat mass. Increased intraabdominal fat is known to contribute to hyperglycemia and hyperinsulinemia due to increased secretion of free fatty acids. These factors are known to affect aortic stiffness. The closer association of waist circumference with visceral fat and cardiovascular risk factors than $\mathrm{BMI}^{29}$ explains 
a
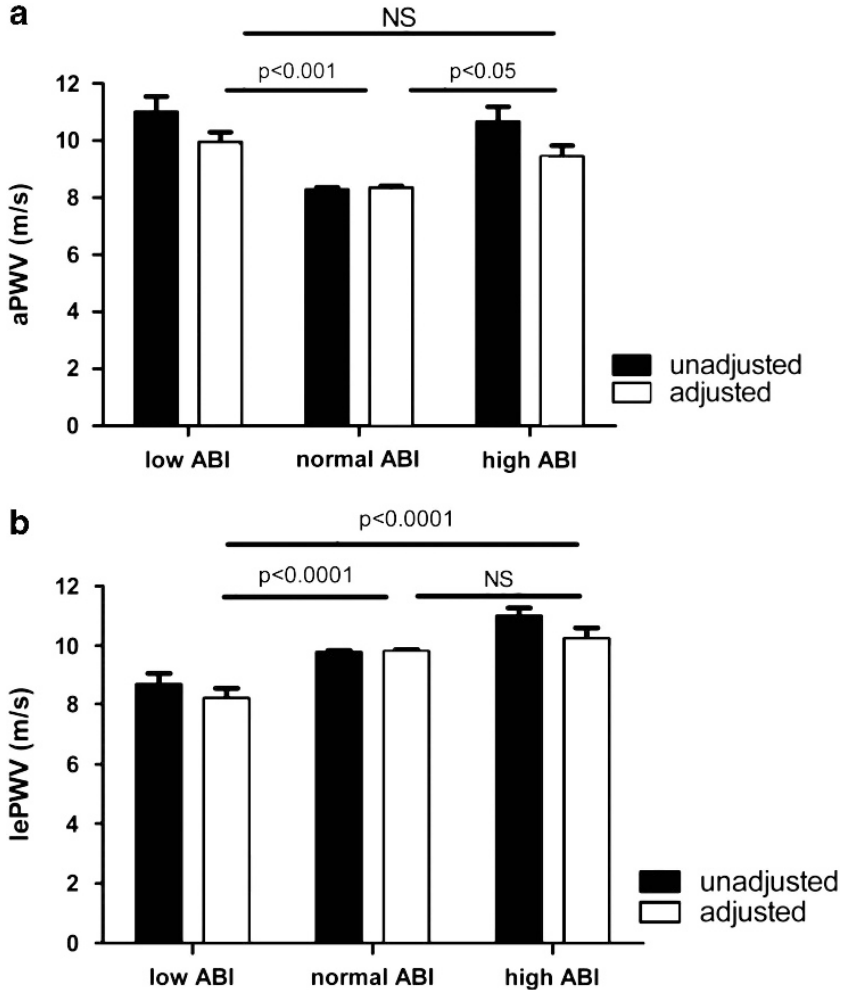

Figure 3 Association of $A B I$ with aPWV (a) and lePWV (b). Data are shown before and after adjustments for age, gender and MAP. Significance values are for adjusted models, see text for $P$-values of unadjusted models. $\mathrm{ABI}$, ankle-brachial index; aPWV, aortic pulse wave velocity; lePWV, lowerextremity pulse wave velocity; MAP, mean arterial pressure. A full color version of this figure is available at the Hypertension Research journal online.
Table 3 Multivariate stepwise analysis of the determinants of aPWV, lePWV and aSBP

\begin{tabular}{lccc}
\hline & Standardized B & $r^{2}$ & $P$ \\
\hline Aortic pulse wave velocity ${ }^{2}, r^{2}=0.55$ & & & \\
Age (years) & 0.404 & 0.365 & $<0.001$ \\
MAP (mm Hg) & 0.109 & 0.108 & $<0.001$ \\
Heart rate (beats per min) & 0.184 & 0.031 & $<0.001$ \\
aSBP (mm Hg) & 0.160 & 0.026 & $<0.001$ \\
Waist circumference (cm) & 0.105 & 0.014 & $<0.001$ \\
bSBP (mm Hg) & 0.137 & 0.010 & $<0.001$ \\
CKD & 0.081 & 0.006 & $<0.001$ \\
& & & \\
Lower-extremity pulse wave velocity ${ }^{2}, \mathrm{r}^{2}=0.21$ & & \\
aSBP (mm Hg) & 0.172 & 0.128 & $<0.001$ \\
Age (years) & 0.181 & 0.022 & $<0.001$ \\
Gender (female) & -0.161 & 0.023 & $<0.001$ \\
MAP (mm Hg) & 0.144 & 0.010 & $<0.001$ \\
Low ABI & -0.104 & 0.011 & $<0.01$ \\
Hip circumference (cm) & -0.081 & 0.006 & $<0.01$ \\
Ankle systolic blood pressure ${ }^{\text {ab }}, \mathrm{r}^{2}=0.62$ & & \\
bSBP (mm Hg) & 0.382 & 0.515 & $<0.001$ \\
MAP (mm Hg) & 0.333 & 0.078 & $<0.001$ \\
aPWV (m s ${ }^{-1}$ ) & 0.114 & 0.018 & $<0.001$ \\
Height (cm) & 0.071 & 0.003 & $<0.001$ \\
Age (years) & 0.092 & 0.004 & $<0.001$ \\
lePWV (m s ${ }^{-1}$ ) & 0.063 & 0.004 & $<0.01$ \\
\hline & & & \\
\hline
\end{tabular}

Abbreviations: $\mathrm{ABI}$, ankle-brachial index; aSBP, ankle systolic blood pressure; aPWV, aortic pulse wave velocity; bSBP, brachial systolic blood pressure; CKD, chronic kidney disease; lePWV, lower-extremity pulse wave velocity; MAP, mean arterial pressure.

${ }^{a}$ Adjusted for antihypertensive and lipid-lowering therapy.

bIndividuals with low $\mathrm{ABI}$ excluded.

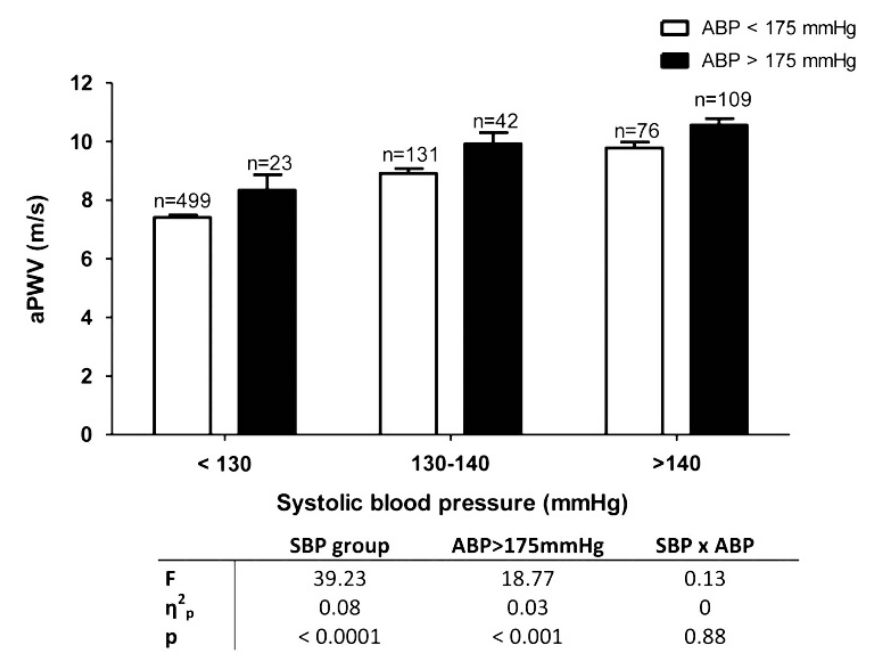

Figure 4 Effect of bSBP and aSBP on aortic pulse wave velocity. aSBP, ankle systolic blood pressure; aPWV, aortic pulse wave velocity; bSBP, brachial systolic blood pressure. why waist circumference was associated with aPWV in multiple regression analysis, while BMI was not. Our finding is in agreement with previous reports ${ }^{30,31}$ in which only waist circumference was independently associated with aortic stiffness, while BMI, hip circumference, fat mass, fat free mass and the waist/hip ratio were not. While waist circumference was associated with increased aPWV, we found increased hip circumference to be linked to lower lePWV. This finding is in agreement with the results of the Hoorn study, in which larger leg fat mass and leg lean mass were associated with lower peripheral arterial stiffness. This may be one of the explanations why increased hip circumference is associated with lower cardiovascular risk in population studies, ${ }^{32}$ and why the gynoid type of obesity is less deleterious than the android one.

Another new finding is the association between aSBP and aPWV that was independent of cardiovascular risk factors including brachial SBP. Furthermore, we found aSBP to be more closely related to aortic stiffness than to lePWV. This suggests that aSBP is more a parameter of aortic stiffness than that of lower-extremity stiffness. Increased transmission of pulsatile energy to the periphery due to increased aortic stiffness may be the potential mechanism explaining association between aSBP and aPWV. ${ }^{33}$ Increased pulsatile stress to the peripheral circulation due to aortic stiffening is caused by loss of buffering function of aorta and impedance mismatch loss between aorta and peripheral circulation. Normally, wave reflection occurs due to arterial stiffness mismatch between the aorta and large muscular arteries. 
Partial wave reflection protects microcirculation from the damaging effect of pulsatile energy. As aortic stiffness meets the stiffness of large muscular arteries, wave reflection is reduced and more pulsatile energy is transmitted to the periphery. Our results suggest that increased aSBP is linked to increased aortic stiffness and may be a parameter of increased pulsatile energy transmission to the periphery. To confirm this theory, energy waves in posterior tibial artery will have to be assessed in the future. The association between aSBP and aortic stiffness may explain the observed positive association between lower-extremity stiffness and LV mass, which is independent of subclinical atherosclerosis. ${ }^{10}$

The independent predictive role of the aSBP in cardiovascular prediction was described previously. In a population study, ${ }^{16}$ aSBP $>175 \mathrm{~mm} \mathrm{Hg}$ was an independent predictor of total and cardiovascular mortality in asymptomatic individuals independently of all traditional cardiovascular risk factors. In another study, aSBP improved the prediction of cardiovascular events independently of classical risk factors. ${ }^{34}$ Further studies will be needed to assess the predictive role of ankle SBP in cardiovascular prediction. The advantage of the aSBP examination is that it is fast, non-invasive, feasible and widely available.

We found decreased lePWV in individuals with lower-extremity PAD (low ABI group). We think that this is an artifact due to significant stenosis. The explanation of this phenomenon can be the blood pressure decrease behind stenosis. Distending pressure is one of the major determinants of arterial stiffness. Moreover, pressure waveform changes have been reported in patients with PAD. Among other things, delay in the foot of the waveform has been reported in patients with PAD compared with normal subjects. ${ }^{35}$ It is the foot of the pulse wave that is commonly used for time delay calculation in order to estimate PWV. Another explanation is the increase in the distance traveled due to collateral circulation. The effect of PAD on brachial-ankle PWV (baPWV) was described previously. Yokoyama et $a .^{36}$ reported baPWV reduction in a leg affected with PAD compared with the non-affected leg. Moreover, baPWV increased following successful stenosis dilatation. In another study, an ABI of 0.95 was calculated to be the cutoff value for diminished accuracy of baPWV. ${ }^{37}$

In the current study, we estimated the aortic length by subtracting the jugular fossa to the carotid pulsation distance from the jugular fossa to the femoral artery pulsation side. This method is known to underestimate the true aortic stiffness. The current consensus for aortic stiffness measurement is to use $80 \%$ of the direct distance between carotid and femoral measurement sides with the cutoff value of $10 \mathrm{~m} \mathrm{~s}^{-1} .14$ However, this has no effect on the strength of association with cardiovascular risk factors and the conclusions of our study.

Our results suggest that conventional cardiovascular risk factors, except for hypertension, have only a small effect on lower-extremity arterial stiffness. The effect of aortic stiffness on peripheral vasculature may be explained by increased pulsatile energy transmission to the periphery due to increased aortic stiffness. Increased ankle systolic blood pressure may be a parameter of increased pulsatile energy transmission to the periphery. Further research is needed to evaluate energy waves in lower-extremity arteries and to confirm the association between increased ankle blood pressure and microcirculation damage.

\section{ACKNOWLEDGEMENTS}

The study was supported by unrestricted research grants from Krka Czech Republic and Servier Czech Republic.
1 Laurent S, Boutouyrie P. Arterial stiffness: a new surrogate end point for cardiovascular disease? J Nephrol 2007; 20 (Suppl 12), S45-S50.

2 Blacher J, Pannier B, Guerin AP, Marchais SJ, Safar ME, London GM. Carotid arteria stiffness as a predictor of cardiovascular and all-cause mortality in end-stage renal disease. Hypertension 1998; 32: 570-574.

3 Barenbrock M, Kosch M, Joster E, Kisters K, Rahn KH, Hausberg M. Reduced arterial distensibility is a predictor of cardiovascular disease in patients after renal transplantation. J Hypertens 2002; 20: 79-84.

4 Dijk JM, Algra A, van der Graaf Y, Grobbee DE, Bots ML. Carotid stiffness and the risk of new vascular events in patients with manifest cardiovascular disease. The SMART study. Eur Heart J 2005; 26: 1213-1220.

5 Pannier B, Guerin AP, Marchais SJ, Safar ME, London GM. Stiffness of capacitive and conduit arteries: prognostic significance for end-stage renal disease patients. Hypertension 2005; 45: 592-596.

6 Tillin T, Chambers J, Malik I, Coady E, Byrd S, Mayet J, Wright AR, Kooner J, Shore A, Thom S, Chaturvedi N, Hughes A. Measurement of pulse wave velocity: site matters. $J$ Hypertens 2007; 25: 383-389.

7 Taniwaki H, Shoji T, Emoto M, Kawagishi T, Ishimura E, Inaba M, Okuno Y, Nishizawa Y. Femoral artery wall thickness and stiffness in evaluation of peripheral vascular disease in type 2 diabetes mellitus. Atherosclerosis 2001; 158: 207-214.

8 Suzuki E, Kashiwagi A, Nishio Y, Egawa K, Shimizu S, Maegawa H, Haneda M, Yasuda $\mathrm{H}$, Morikawa S, Inubushi T, Kikkawa R. Increased arterial wall stiffness limits flow volume in the lower extremities in type 2 diabetic patients. Diabetes Care 2001; 24: 2107-2114.

9 Kizu A, Koyama H, Tanaka S, Maeno T, Komatsu M, Fukumoto S, Emoto M, Shoji T, Inaba M, Shioi A, Miki T, Nishizawa Y. Arterial wall stiffness is associated with peripheral circulation in patients with type 2 diabetes. Atherosclerosis 2003; 170: 87-91.

10 Ix JH, Katz R, Peralta CA, e Boer IH, Allison MA, Bluemke DA, Siscovick DS, Lima JA, Criqui $\mathrm{MH}$. A high ankle brachial index is associated with greater left ventricular mass MESA (Multi-Ethnic Study of Atherosclerosis). J Am Coll Cardiol 2010; 55: 342-349.

11 Cifkova R, Skodova Z, Bruthans J, Adamkova V, Jozifova M, Galovcova M, Wohlfahrt P, Krajcoviechova A, Poledne R, Stavek P, Lanska V. Longitudinal trends in major cardiovascular risk factors in the Czech population between 1985 and 2007/8. Czech MONICA and Czech post-MONICA. Atherosclerosis 2010; 211: $676-681$.

12 Levey AS, Stevens LA, Schmid CH, Zhang YL, Castro AF 3rd, Feldman HI, Kusek JW, Eggers P, Van Lente F, Greene T, Coresh J. A new equation to estimate glomerular filtration rate. Ann Intern Med 2009; 150: 604-612.

13 Wohlfahrt P, Palous D, Ingrischova M, Krajcoviechova A, Seidlerova J, Galovcova M, Bruthans J, Jozifova M, Adamkova V, Filipovsky J, Cifkova R. A high ankle-brachial index is associated with increased aortic pulse wave velocity: the Czech post-MONICA study. Eur J Cardiovasc Prev Rehabil 2011; 18: 790-796.

14 Van Bortel LM, Laurent S, Boutouyrie P, Chowienczyk P, Cruickshank JK, De Backer T, Filipovsky J, Huybrechts S, Mattace-Raso FU, Protogerou AD, Schillaci G, Segers P, Vermeersch S, Weber T. Expert consensus document on the measurement of aortic stiffness in daily practice using carotid-femoral pulse wave velocity. J Hypertens 2012; 30: 445-448.

15 Wohlfahrt P, Ingrischova M, Krajcoviechova A, Palous D, Dolejsova M, Seidlerova J, Galovcova M, Bruthans J, Jozifova M, Adamkova V, Filipovsky J, Cifkova R. A novel oscillometric device for peripheral arterial disease screening in everyday practice. The Czech-post MONICA study. Int Angiol 2011; 30: 256-261.

16 Hietanen $\mathrm{H}$, Paakkonen R, Salomaa V. Ankle blood pressure as a predictor of total and cardiovascular mortality. BMC Cardiovasc Disord 2008; 8: 3.

17 Laurent S. Arterial wall hypertrophy and stiffness in essential hypertensive patients. Hypertension 1995; 26: 355-362.

18 Laurent S, Boutouyrie P, Lacolley P. Structural and genetic bases of arterial stiffness. Hypertension 2005; 45: 1050-1055.

19 Boutouyrie P, Laurent S, Benetos A, Girerd XJ, Hoeks AP, Safar ME. Opposing effects of ageing on distal and proximal large arteries in hypertensives. J Hypertens Supp/ 1992; 10: S87-S91.

20 Benetos A, Laurent S, Hoeks AP, Boutouyrie PH, Safar ME. Arterial alterations with aging and high blood pressure. A noninvasive study of carotid and femoral arteries. Arterioscler Thromb 1993; 13: 90-97.

21 Emoto M, Nishizawa Y, Kawagishi T, Maekawa K, Hiura Y, Kanda H, Izumotani K, Shoji $\mathrm{T}$, Ishimura $\mathrm{E}$, Inaba M, Okuno $\mathrm{Y}$, Morii $\mathrm{H}$. Stiffness indexes beta of the common carotid and femoral arteries are associated with insulin resistance in NIDDM. Diabetes Care 1998; 21: 1178-1182.

22 Lo CS, Relf IR, Myers KA, Wahlqvist ML. Doppler ultrasound recognition of preclinical changes in arterial wall in diabetic subjects: compliance and pulse-wave damping. Diabetes Care 1986; 9: 27-31.

23 Blacher J, Demuth K, Guerin AP, Safar ME, Moatti N, London GM. Influence of biochemical alterations on arterial stiffness in patients with end-stage renal disease. Arterioscler Thromb Vasc Biol 1998; 18: 535-541.

24 Kimoto E, Shoji T, Shinohara K, Inaba M, Okuno Y, Miki T, Koyama H, Emoto M, Nishizawa Y. Preferential stiffening of central over peripheral arteries in type 2 diabetes. Diabetes 2003; 52: 448-452.

25 Laurent S, Girerd X, Mourad JJ, Lacolley P, Beck L, Boutouyrie P, Mignot JP, Safar M. Elastic modulus of the radial artery wall material is not increased in patients with essential hypertension. Arterioscler Thromb 1994; 14: 1223-1231. 
26 Mourad JJ, Girerd X, Boutouyrie P, Safar M, Laurent S. Opposite effects of remodeling and hypertrophy on arterial compliance in hypertension. Hypertension 1998; 31 (1 Pt 2), 529-533.

27 Filipovsky J, Ticha M, Cifkova R, Lanska V, Stastna V, Roucka P. Large artery stiffness and pulse wave reflection: results of a population-based study. Blood Press 2005; 14 : 45-52.

28 Vermeersch SJ, Rietzschel ER, De Buyzere ML, De Bacquer D, De Backer G, Van Borte LM, Gillebert TC, Verdonck PR, Segers P. Age and gender related patterns in carotidfemoral PWV and carotid and femoral stiffness in a large healthy, middle-aged population. J Hypertens 2008; 26: 1411-1419.

29 Janssen I, Katzmarzyk PT, Ross R. Waist circumference and not body mass index explains obesity-related health risk. Am J Clin Nutr 2004; 79: 379-384.

30 Czernichow S, Bertrais S, Oppert JM, Galan P, Blacher J, Ducimetiere P, Hercberg S, Zureik M. Body composition and fat repartition in relation to structure and function of large arteries in middle-aged adults (the SU.VI.MAX study). Int J Obes (Lond) 2005; 29: 826-832.

31 Schillaci G, Pirro M, Vaudo G, Mannarino MR, Savarese G, Pucci G, Franklin SS, Mannarino E. Metabolic syndrome is associated with aortic stiffness in untreated essential hypertension. Hypertension 2005; 45: 1078-1082.
32 Snijder MB, Dekker JM, Visser M, Yudkin JS, Stehouwer CD, Bouter LM, Heine RJ, Nijpels G, Seidell JC. Larger thigh and hip circumferences are associated with better glucose tolerance: the Hoorn study. Obes Res 2003; 11: 104-111.

33 Mitchell GF. Effects of central arterial aging on the structure and function of the peripheral vasculature: implications for end-organ damage. J App/ Physiol 2008; 105 1652-1660.

34 Hietanen HJ, Paakkonen R, Salomaa V. Ankle blood pressure and pulse pressure as predictors of cerebrovascular morbidity and mortality in a prospective follow-up study. Stroke Res Treat 2011; 2010: 729391.

35 Kempczinski RF. Segmental volume plethysmography in the diagnosis of lower extremity arterial occlusive disease. J Cardiovasc Surg (Torino) 1982; 23. 125-129.

36 Yokoyama H, Shoji T, Kimoto E, Shinohara K, Tanaka S, Koyama H, Emoto M, Nishizawa $Y$. Pulse wave velocity in lower-limb arteries among diabetic patients with peripheral arterial disease. J Atheroscler Thromb 2003; 10: 253-258.

37 Motobe K, Tomiyama H, Koji Y, Yambe M, Gulinisa Z, Arai T, Ichihashi H, Nagae T, Ishimaru S, Yamashina A. Cut-off value of the ankle-brachial pressure index at which the accuracy of brachial-ankle pulse wave velocity measurement is diminished. Circ J 2005; 69: 55-60. 